\title{
Microcomputer requirements in the operational Navy
}

\author{
MELVYN C. MOY \\ Navy Personnel Research and Development Center, San Diego, California
}

\begin{abstract}
The microcomputer seems destined to play an ever increasing role in today's operational Navy. It promises solutions to problems ranging from inventory control to tactical decision making. With that promise, however, comes an inevitable array of new problems and questions to be solved. The Navy constantly must evaluate systems on the basis of the systems' ability to produce immediate gains. Design characteristics most important in the choice of systems include application software, equipment reliability and availability, software maintainability, ease of use, and integrated approach. The Navy's demand for more properly human-engineered systems far exceeds that of industry in general. Until designers can deliver such systems, the influx of microcomputers into the operational Navy will continue to be thought of as an unnecessary proliferation.
\end{abstract}

Over the last several years, microcomputers have been appearing aboard Navy ships in increasing numbers. Originally, they were brought aboard as stand-alone word processors, as part of microcomputer-based training projects, or as aids in analyzing meteorologic and tactical data. More often than not, the programs were those developed by defense contractors or Navy laboratories, rather than by shipboard personnel. As the costs for microcomputers decreased, members of ships' companies began bringing their own computers aboard for personal and hobbyist use. It was not long before people began developing microcomputer applications to support daily operations and reporting requirements that heretofore had been done manually and had consumed a significant portion of time. Many of these applications were adopted by individual ships as being extremely beneficial in saving man-hours of labor and helping the ships to maintain high levels of readiness. Application programs were developed mainly to support ships in nontactical areas, such as personnel and financial management, inventory control, maintenance scheduling, and shipboard security. The programs were tailored basically to match the operating procedures of a particular ship and the style of the people actively involved in the input and use of the information being managed. Typically, the functions were developed and operated by the owner of the microcomputer and had little or no documentation. There was seldom any attempt to link related applications so that the same information might be shared by different application modules. If the various modules were integrated or shared any consistency in design, it was an

The opinions expressed here are solely those of the author and are not to be construed as those of the Department of the Navy or the Department of Defense. The author's mailing address is: Navy Personnel Research and Development Center, San Diego, CA 92152. artifact of the individual doing the development. One must appreciate the circumstances under which these applications were born and developed. It is not hard to understand that the computer cognoscenti were but a small group, seldom numbering more than two on any ship. Although the military does have people trained to be data processors (DP) or data specialists (DS), these ratings are intended to cover individuals who work with batch-mode mainframes or minicomputers. Their skills and knowledge do not transfer comple tely to the microcomputer environment. This has been a common experience in the data processing divisions of private industry as well. Thus, the sailor with the talent, ability, and opportunity to develop these applications on microcomputers is indeed a rare commodity. Unfortunately, the military has a penchant for regularly moving people between assignments. When the sailor with the microcomputer leaves a shipboard assignment, so does his microcomputer and, effectively, the applications for which he was responsible. For some ships, this loss posed serious problems, since the ships had become quite dependent upon this newly acquired capability and really had no direct way of replacing it. The acquisition of hardware, software, and properly trained personnel to do the job with microcomputers was not exactly established procedure. Even when equipment could be made available, the lack of adequate documentation, the idiosyncratic nature of the software design, and the unlikelihood of obtaining replacement personnel of equivalent facility with microcomputers made the situation difficult, if not impossible. The solution to this problem was not to cease and desist in the use of microcomputers aboard ships. The gains and advantages in using microcomputers were too great to give up, despite painful repetitions of the problems just mentioned. The use of microcomputers had to become a formally recognized procedure in the Navy so that: (1) uniform standards could be set for system con- 
figurations to promote transportability of applications software; (2) quality control and documentation requirements could be set;(3) redundant development of software could be reduced or eliminated; (4) a pipeline could be established to provide a sufficient number of qualified personnel to support the ships in the fleet; (5) a central authority could be given the responsibility for providing software support to all the ships; and most important, (6) a funding line could be established to provide for all of the above.

The Navy, as is the military in general, is currently in the midst of making all these things come true. There has been an enormous increase in the number of authorized purchases of microcomputers. The Navy and the Air Force have recently awarded a contract for 10,000 single-user desktop computer systems. Assuming, conservatively, that half of these units will go aboard ships in the fleet, that means an average of almost 10 microcomputers for each ship from this purchase alone. Add to that the number of word processors and stand-alone microcomputers placed aboard ships in the past for one purpose or another, and one can see that there will soon be a strikingly large population of microcomputer systems in the fleet. At some point, the concern for proliferation of computer hardware becomes an issue. Each additional request or acquisition increases the opposition to the next request, much like the process of reactive inhibition. The general utility and cost effectiveness of microcomputers are still very much suspect, despite claims to the contrary. The units in the fleet now and those that will be there in the near future are being looked upon as a long-term experiment and not necessarily as the use of a proven technology-with the possible exception of the word processing function. In order for the experiment to be successful, positive gains attributable to such systems must be demonstrated quantitatively across several dimensions, such as improved mission capability, reductions in manning requirements, improvement in individuals' skills, and greater job satisfaction. Shipboard microcomputer systems will have to avoid the pitfalls and hidden costs that many people fear are inevitable.

The intended typical user in the fleet will have little or no formal training in the use of computer systems. The career paths and background of sailors are considerably different from those of typical white-collar workers who use computer systems in the private sector. Therefore, the design of hardware and software systems for use in the fleet must place a minimum requirement for any involved training and prerequisite knowledge. The interface between users and the computer system must be mastered easily and be as uncomplicated as possible for the naive user. Yet there must be a readily invoked means for experienced users to circumvent these more time-consuming, explicit interfaces in favor of direct command dialogues.

It must be remembered that the typical microcomputer user in the Navy will only be doing so part-time, since each person has several other major and primary responsibilities when it comes to conducting warfare. Thus, it is unlikely that these individuals will have as much time and opportunity as users in the civilian sector to master the use of microcomputer systems. The individual in the Navy is time-sharing across several different jobs. Because of this fact, the design of computer systems for the shipboard user takes on many of the same requirements as the design of decision support systems for executives (Bennett, 1983). As such, considerable attention must be given to the human engineering of the system to accommodate what information is known about the cognitive functioning and limitations of a user interacting with a computer.

The Navy Personnel Research and Development Center (NPRDC) has been involved in the evaluation of an experimental system of networked microcomputers aboard the nuclear carrier U.S.S. Carl Vinson. The system consists of 27 PERQ computers connected through an Ethernet local area network and supports ZOG as developed by researchers at Carnegie-Mellon University (Robertson, McCracken, \& Newell, 1981). ZOG is an environment that serves as an operating system, as a user interface, and as a hierarchical information storage and retrieval system. Data exist in the computer as viewable screen frames that are linked together into nets and subnets. The user moves around in the ZOG environment by accessing a succession of screen frames, starting at some general high-level entry point and working down toward greater specificity. Each frame has a formal structure and can contain information in narrative text form with menu options to select additional frames of information. Theoretically, the user continues to select the menu options most appropriate for his or her requirements and sees successive frames of information, each being more specific than the one before it, until a level of granularity has been reached at which the individual has either found the information he or she seeks or can determine that the information does not exist in the database. The user can rapidly move forward or backward through the information paths and can place markers along the way to establish reference points to which he or she might retreat if he or she should become lost in one of the subnets. The user has the ability to expand on-screen frames or to extend the information network by developing additional frames and linking them to the existing ones. Any frame in the ZOGnet can be linked to any other frame simply by using an option that automatically establishes pointers within the computer to accomplish that. These connections can be made and broken at any time without the user's having to understand database structures. To avoid potential disaster, however, there is a security protection against the unauthorized alteration of major frames and linkages in the ZOG system.

The user may select options known as agents. Agents are actually programs that can be invoked to perform a myriad of functions, such as editing, printing, calcula- 
tions, etc. The establishment of the linking pointers mentioned above is done through an agent. Agents can be thought of as the tools or operators for decision support systems, as discussed by Bennett (1983).

The networking of microcomputers makes it possible to share information or frames, to create a total ZOG environment that would be much too large to be accommodated on any single machine, and to achieve the redundancy necessary for sustained operations. The PERQ computers themselves were distributed throughout the ship in areas where it was felt there would be the most usage and benefit. Users of the system can monitor the current value of changing information or computer results based on that information at the same time the information is being entered. For example, the captain on the bridge can monitor the projected consequence upon aircraft recovery due to an emergency landing of a plane with low fuel if such changes might affect tactical or navigational decisions.

Because it is based upon a menu-selection user interface, the ZOG system is intrinsically easy for most people to use with only a minimal amount of training. Since the ZOG system is quite generic and can be used for several different things, it was anticipated that the ZOG system would grow rapidly over time as users discovered its characteristics and how they might develop ZOG subnets to support them in the performance of their responsibilities. Department heads on the ship were encouraged by the commanding officer to procedurize their job functions and to attempt to develop ZOG subnets to instantiate these procedures. The benefits were expected to be at least threefold. First, it was hoped that the act of analyzing their responsibilities in order to procedurize them would produce insights about inefficiencies or shortfalls and thus make them more aware of how to perform their duties better. Second, the information produced would become apparent and available to others who had to interact and coordinate their activities, thus producing better teamwork and appreciation for each other aboard the ship. Third, the instantiated information is readily available as corporate memory and reduces the start-up and familiarization problems normally associated with change in personnel aboard the ship.

To facilitate the evaluation of the ZOG system aboard the U.S.S. Carl Vinson while the ship was at sea for 8 months, a ZOG agent was developed to automatically monitor and record user performance data. The information was collected from each machine on a regular basis and placed on floppy disks that were mailed back to our laboratory for analysis. The data consisted of in . formation about the user identification (ID), date and time of use, ZOG subnets accessed and time spent in each, response times to frames, illegal entries, and agents invoked. Although we have not received or analyzed all the data from the machines as of this date, we do have preliminary results that emphasize the importance of perceived system availability and reliability, on-line tu- torials, quick reference aids, and support staff to provide user assistance. During the first part of the ship's cruise, the ZOG system was being used by only a small group of the people who received training. This was despite the fact that occasional users, including many people who had received no training except exposure to the on-line tutorial, demonstrated that they could use the ZOG system quite well. We later discovered that the users were often experiencing problems with random system crashes that proved to be more a nuisance than a fatal problem. Although data seldom were lost in these events, the users equated the events with system unreliability and began to doubt the validity of the data contained. Once the problem was alleviated through a quick fix, system usage increased by $50 \%$ almost immediately. As the application subnets began to be developed, discretionary users found greater utility in the system and soon found themselves adding to the existing subnets.

A Navy ship presents an impossible situation for anyone attempting to develop ergonomically sound workstations. The best advice that can be given is to try to make everything adjustable, because the equipment will probably have to adapt to the workplace. It is interesting to note that none of the 28 machines were ever relocated because of inadequate working conditions. Machines were moved, but only because more use of the system could be made at a different location. It is enlightening to note that in most cases the system was being used extensively despite these negative factors.

Under the circumstances, it was not possible to systematically control conditions to determine whether even greater use could be obtained under more ideal conditions or whether there are any long-term detrimental effects. The ZOG system being evaluated is an evolving system that was moved directly from the university laboratory into an operational environment-an unusual event. Nonetheless, it provides an excellent opportunity to examine the feasibility and advisability of implementing certain features with microcomputers on board a Navy ship. When microcomputers are woven together in a network in the way in which it was done in the $\mathrm{ZOG} /$ Vinson project, the user receives additional benefits in having a consistent interface with the system across a wide range of applications. The use of a network gives the appearance of a central source for storage and retrieval of information, thus eliminating redundant data entry. Yet the network permits decentralized processing of the information, providing speed and redundancy over time-shared systems.

Ironically, one of the intents of the large contract for microcomputers is to ascertain what applications along with what approaches to those applications are desirable so that they can be institutionalized as part of the formal Navy program to provide ships with automated administrative and management capabilities, the Shipboard Nontactical ADP Programs (SNAP I and SNAP II). These two programs are based upon the use of minicom- 
puters with several distributed terminals running in timesharing mode. They do not provide quite the same latitude for shipboard development work as do microcomputers, and they do not make a very good host for the computer-based instruction packages that are being discussed in this session.

\section{REFERENCES}

BENNETT, J. L. (Ed.). (1983). Building decision support systems. Reading, MA: Addison-Wesley.

Robertson, G., McCracken, D., \& Newell, A. (1981). The ZOO approach to man-machine communication. International Journal of Man-Machine Studies, 14, 461-488. 